Revue musicale OICRM

\title{
De Shakespeare à Diam's en passant par George Lucas et PNL. Kenny Thomas, intervenant social et médiateur
}

\section{Elsa Fortant}

Volume 7, numéro 2, 2020

URI : https://id.erudit.org/iderudit/1072418ar

DOI : https://doi.org/10.7202/1072418ar

Aller au sommaire du numéro

\section{Éditeur(s)}

Observatoire interdisciplinaire de création et recherche en musique (OICRM)

\section{ISSN}

2368-7061 (numérique)

Découvrir la revue

\section{Citer ce document}

Fortant, E. (2020). De Shakespeare à Diam's en passant par George Lucas et PNL. Kenny Thomas, intervenant social et médiateur. Revue musicale OICRM, 7(2), 101-110. https://doi.org/10.7202/1072418ar
Résumé de l'article

Après s'être évertuées à démocratiser l'accès à la culture, les politiques culturelles développées au Québec et en France depuis la fin des années 1980 mettent l'emphase sur les pratiques amateurs et sur la participation citoyenne. Pour rejoindre ces publics et dans des objectifs d'émancipation des populations, les programmes d'action culturelle ont recours à la médiation culturelle, souvent définie comme une approche qui permet de faire le pont entre des pratiques artistiques et des publics. À travers le parcours de Kenny Thomas, travailleur social qui est aussi musicien et rappeur, cet article met en lumière les liens qui existent entre les différents champs auxquels sa pratique se rattache, autrement dit l'intervention sociale et la médiation. Il démontrera que les frontières entre ces mondes sont malléables au point parfois de se confondre. 


\title{
De Shakespeare à Diam's en passant par George Lucas et PNL. Kenny Thomas, intervenant social et médiateur
}

\author{
Elsa Fortant
}

\begin{abstract}
Résumé
Après s'être évertuées à démocratiser l'accès à la culture, les politiques culturelles développées au Québec et en France depuis la fin des années 1980 mettent l'emphase sur les pratiques amateurs et sur la participation citoyenne. Pour rejoindre ces publics et dans des objectifs d'émancipation des populations, les programmes d'action culturelle ont recours à la médiation culturelle, souvent définie comme une approche qui permet de faire le pont entre des pratiques artistiques et des publics. À travers le parcours de Kenny Thomas, travailleur social qui est aussi musicien et rappeur, cet article met en lumière les liens qui existent entre les différents champs auxquels sa pratique se rattache, autrement dit l'intervention sociale et la médiation. Il démontrera que les frontières entre ces mondes sont malléables au point parfois de se confondre.
\end{abstract}

Mots clés : médiation de la musique ; musiques pop ; modèle participatif ; Kenny Thomas ; récit d'expérience.

\begin{abstract}
After striving to democratize access to culture, cultural policies developed in Quebec and France since the end of the 1980's have focused on amateur practices and citizen participation. To reach these audiences and with the aim of empowering populations, cultural action programs resort to cultural mediation, often defined as an approach that allows for bridging between artistic practices and audiences. Through the experience of Kenny Thomas, a social worker who's also a musician and rapper, this article highlights the existing relationship between the fields to which his practice relates, social intervention and mediation. It will demonstrate that the boundaries between worlds are malleable, to the point of sometimes being blurred.

Keywords: participatory model; popular music; music mediation; Kenny Thomas; working experience.
\end{abstract}




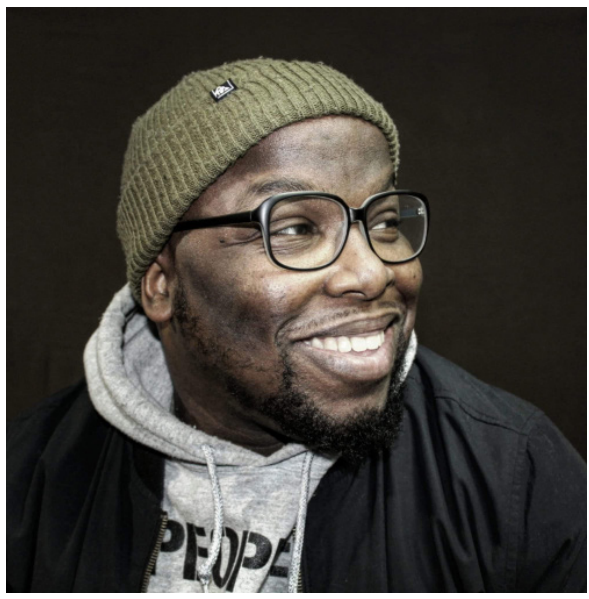

Figure 1 : Kenny Thomas par lui-même, 2019.

Montréalais d'origine haïtienne, né à Montréal Nord (un quartier identifié comme " défavorisé ${ }^{1}$ " par les politiques publiques montréalaises), Kenny Thomas œuvre comme intervenant social auprès des jeunes depuis plus de 15 ans. Le trentenaire fait partie de ces gens qui impressionnent par leur proactivité et leur facilité à s'adapter à tous types de publics et d'environnements (des maisons de jeunes aux organismes multiculturels). Coach d'impro, il est aussi artiste hip-hop qui écrit, compose, enregistre, arrange, mixe et masterise sa musique, sous le nom de Simon 16.

Kenny Thomas a le contact facile et partage avec plaisir ses expériences et son parcours. Dans le cadre d'un projet comme Samplicité volontaire, que nous vous présenterons dans cette entrevue, il intègre avec brio sa pratique artistique musicale personnelle à sa pratique de médiateur et réciproquement, brouillant ainsi les frontières entre intervention sociale et médiation culturelle.

INTERVENTION SOCIALE, MÉDIATION : D'UNE PRATIQUE À L'AUTRE, IL N'Y A QU'UN PAS

Elsa Fortant : Quelles sont les formes de médiation que tu pratiques ?

Kenny Thomas : Le cégep du Vieux Montréal m'a engagé pour donner des ateliers d'écriture hip-hop. Là, j'ai proposé un cours sur le hip-hop, je vais amener mes machines (boîte à rythmes, échantillonneur). Dans ce cas, l'idée c'est d'aller plus loin. Aborder l'écriture, oui, mais aussi le contexte social dans lequel ça s'inscrit : pourquoi le hip-hop parle souvent des mêmes thématiques, pourquoi la précarité, la pauvreté sont des thèmes centraux de cette musique-là. Il y a un contexte social et historique qui explique ça. À travers cette approche, on touche le rapport à soi-même, le sentiment d'accomplissement. Le hip-hop avec lequel moi j'ai évolué, c'est la musique qui parle beaucoup d'une population un peu mise de côté, marginalisée. C'est une thématique qui revient beaucoup chez les jeunes, ils sont vraiment en recherche d'identité. Souvent quand ils sont en groupe on les voit un peu comme de la racaille, des gens qui vagabondent, qui n'ont pas leur place dans l'espace public.

Je coache de l'impro depuis 2003, par exemple dans les foyers de jeunes travailleurs. Ils ont chacun leur chambre, mais ils doivent partager des heures communes. Souvent, le partage des heures communes cause des frictions ; donc j'essaye d'amener l'activité d'impro de façon ludique, avec humour et légèreté.

1 Voir Ville de Montréal, Plan d'action collectif. Priorité jeunesse 2017-2027, http://ville.montreal.qc.ca/ pls/portal/docs/PAGE/ARROND MNO FR/MEDIA/DOCUMENTS/PLANDACTIONJEUNESSE FINAL WEB 2.PDF, consulté le 19 janvier 2020. 
Le théâtre d'improvisation est une pratique artistique populaire au Québec. Elle s'épanouit aussi bien au sein d'établissements d'enseignement postsecondaires comme les cégeps (collège d'enseignement général et professionnel) et les universités, que dans des cafés-théâtres. Les participants se regroupent à travers des ligues (une forme équivalente à celle d'une troupe) qui s'affrontent lors de matchs d'impro.

Extrait vidéo 1 : "National d'Impro 2017 - FINALE : Lanaudière contre Saguenay Lac-Saint-Jean ».

Voir la vidéo sur YouTube.

Kenny Thomas se sert de l'impro comme un dispositif de médiation pour aborder de manière ludique des questions de « résolution de conflits, de communication saine et de facultés d'adaptation ». À travers le jeu d'acteur, les participants s'expriment et projettent leurs comportements ou réactions sur des simulations de situations issues de la vie quotidienne : entrevues, accusation de vol, situation de harcèlement sexuel au travail. «Le but c'est de former les jeunes afin qu'ils soient outillés pour se trouver un emploi et le garder. Y'a toujours un retour de la part des participants comme « OK, comment est-ce que vous auriez fait ça ? ", " qu'est-ce que vous auriez fait dans la vraie vie » et de les mettre en lien avec des ressources que ce soit les normes du travail, la commission des droits à la personne.

к. т. : Dans un autre contexte, j'ai donné des ateliers d'éducation sexuelle à des garçons, il y a quelques années, et j'utilisais aussi l'humour comme levier. Je leur ai dit : "Je ne suis pas votre prof, pas votre parent, pas votre pasteur, on va parler de sexe, sortez-moi tous les termes qui vous passent par la tête. " Donc là, ils ont été déstabilisés. C'est important d'en parler avec quelqu'un qui ne vous juge pas, souvent vous avez des questions qui peuvent, si on n'y répond pas, changer votre vie, souvent pas nécessairement de façon positive. J'organisais aussi des ateliers de cuisine avec des garçons, ça permettait déconstruire le rapport de genre par rapport aux rôles dans la maison.

E. F. : Entre le métier d'intervenant social, celui d'artiste, de médiateur... comment toutes ces pratiques s'articulent-elles? Comment se nourrissent-elles entre elles?

K. T. : J'ai beaucoup travaillé dans des organismes communautaires, donc avec très peu de moyens financiers, mais ce qui est intéressant dans cet environnement, c'est que les gens viennent sur une base volontaire. Contrairement au centre jeunesse où les personnes présentes le sont à la suite d'une mesure judiciaire. Ce que j'ai appris très tôt dans l'intervention, c'est qu'on peut utiliser n'importe quel prétexte pour rentrer en contact avec des gens. Il y en a pour qui c'est le sport, c'est l'exemple classique. Comme un coach qui intervient auprès des jeunes pour travailler l'esprit d'équipe, la communication, ce genre de choses. J'utilise beaucoup le hip-hop parce que c'est ce que je connais, la musique en général, pour essayer d'aller chercher des gens autour de thématiques bien précises. L'hiver dernier je m'occupais d'un club culturel pour une école d'adultes à Montréal-Nord. Le but du projet c'était d'encourager les jeunes à la lecture, sauf que c'est une école où les jeunes ont à peu près tous des difficultés au niveau académique. Si on aborde le club de lecture comme un club autour du livre, on va les perdre. J'ai développé l'idée d'appeler ça un club culturel. C'est vraiment de 
prendre leurs forces, leurs expertises musicales ou peu importe. Tu sais, même sur les réseaux sociaux, il y a beaucoup de choses, c'est de la lecture aussi... Donc c'était de prendre ces éléments-là et d'essayer d'amorcer un sujet $x$, d'apporter des éléments en lien avec eux.

E. F. : Peux-tu me donner des exemples d'activités réalisées dans ce cadre?

к. т. : Bien sûr. J'ai pris des morceaux de textes d'Oxmo Puccino ${ }^{2}$ versus Denis Diderot. Je prenais juste des extraits sans leur dire de qui c'était et là je leur demandais : « OK, lequel est un rappeur, lequel est un auteur du XVII ${ }^{\mathrm{e}}$ siècle ? » Il y a des vidéos sur Internet où des philosophes décortiquent des paroles de rappeurs ${ }^{3}$ et je trouvais ça cool comme idée, donc j'ai essayé de reprendre ça pour amener le principe de néologisme versus archaïsme, par exemple. Ça peut paraître drôle, mais disons qu'avec Othello [Shakespeare, Verdi] et "Confessions nocturnes » de Diam's ${ }^{4}$, il y a des thèmes qui se recoupent : la trahison, suivre son instinct... Personnellement le hip-hop et le rap ça m'a vraiment raccroché à l'école pour toutes sortes de raisons ; c'est à 17-18 ans que le monde a commencé à avoir une certaine logique pour moi. C'est ce que j'essaye de mettre de l'avant en utilisant la musique comme moyen de rentrer en contact, ou les textes.

Extrait vidéo 2 : "Philo ou Ghetto : qui de IAM ou Nietzsche a dit cette phrase?». Voir la vidéo sur YouTube.

к. т. : J'ai des jeunes qui font de la musique ou qui trippent sur un certain type de musique et quand tu leur apprends que " Timber » (2013) de Kesha ${ }^{5}$ échantillonne "San Francisco Bay » de Lee Oskar ${ }^{6}$, un dude qui a sorti un album en 1979, ils sont super étonnés. Donc à ce moment-là, il faut comprendre comment les personnes avec qui tu entres en contact font des liens elles aussi. Un jeune qui tripe sur une chanson parce que le message vient le chercher, il faut respecter ça et c'est un bon moyen d'ouvrir une porte vers autre chose en posant des questions : "Tu te sens comme ça, mais pourquoi? Est-ce qu'il y a une situation dans ta famille, au travail, à l'école, dans tes relations amoureuses, avec tes amis proches...?» Là, tu développes autre chose.

2 Oxmo Puccino est un rappeur français qu'on rattache à la mouvance du « rap conscient». Ses textes, poétiques, traitent de thématiques sociales, économiques, politiques qui reflètent la réalité des personnes issues de l'immigration.

3 Des philosophes comme Benjamine Weill ou Raphaël Enthoven se sont prêtés à l'exercice d'analyser les punchlines de rappeurs francophones comme Vald, Damso, Booba. Les vidéos sont disponibles sur YouTube. Exemple : Punchlife de Vald vues par la philosophie, https://youtu.be/f9zBw9U6F4w, consulté le 19 janvier 2020.

4 Diam's est une rappeuse française qui a remporté une victoire de la musique en 2004 pour son album Brut de femme. Elle est la rappeuse française qui a connu le plus de succès, avec des disques d'or et de platine. Son rap aborde des thématiques en lien avec la condition féminine, les relations hommes-femmes, l'amour. Elle a été impliquée dans des projets humanitaires comme le Big Up Project qui s'engage pour les enfants défavorisés d'Afrique.

$5 \quad$ Kesha est une chanteuse pop américaine qui s'inspire de la musique des années 1980. Elle a expérimenté la country, le pop-rock et se concentre aujourd'hui à rapper sur des musiques électroniques.

6 Lee Oskar est un joueur d'harmonica danois connu notamment pour avoir participé au groupe de fusion rock-funk War des années 1970 à 1990. Il a poursuivi une carrière solo et a aujourd'hui sa propre marque d'harmonicas. 
Kenny Thomas utilise la médiation culturelle comme un outil dans sa pratique d'intervenant social, en prenant pour objet la musique, comme le rap. Cela lui permet de nouer le contact avec les jeunes et surtout de les placer au cœur du dispositif en partant de leur expérience. Il s'inscrit dans une démarche en lien avec la démocratie culturelle $^{7}$. Pour cette raison, les rapports humains qui sont développés par la suite avec les participants tendent vers l'horizontalité. Le modèle mis en avant ici s'éloigne de celui, très vertical, de l'éducation populaire - même si l'apprentissage est une dimension importante dans les divers dispositifs de médiation mis en place par Kenny Thomas - et des visions en lien avec la démocratisation culturelle (toujours très verticale).

E. F. : En t'écoutant parler, on a l'impression que de ton point de vue, médiation culturelle et intervention sociale sont interreliées...

к. т. : Oui toujours, mais pas forcément de façon consciente. Les jeunes de 16-25 ans ont davantage besoin d'une personne disponible ; l'écoute va venir par la suite. Je travaille beaucoup avec les jeunes du quartier du Nord-Est et il y a des dynamiques assez particulières, à 16-17 ans ; même à 20-21 ans, le rapport à l'adulte est toujours négatif. C'est un parent, un professeur, la police, un intervenant des centres jeunesse, un juge... Ce qui fait que quand tu arrives avec l'idée "Ah oui, toi aussi t'aimes Youssoupha $^{8}$ ", tu échanges. Le rapport n'est plus le même, pour ce jeune-là, devant lui, il a une personne qui a un intérêt pour ce qu'il est et non pas pour ce qu'il représente. Entre intervenants on se dit souvent ça. On voit souvent les jeunes ou les populations avec qui on est appelés à travailler comme des "problématiques ». Souvent un jeune, c'est comme Robocop (tu vois la description sur le côté-là et tout), mais quand c'est pas du tout ton approche pour rentrer en contact avec les jeunes, c'est autre chose.

Récemment, j'ai quitté un emploi que j'ai eu pendant quatre ans et j'ai des jeunes qui me textent encore pour me dire qu'ils se sont trouvés une job. On a développé autre chose. Je ne suis pas un adulte qui leur dit quoi faire. Ça n'empêche pas que je vais les remettre en question si je trouve qu'ils ont des choix de vie qui sont questionnant ou quand je vois qu'ils stagnent, mais jamais j'ai cette relation d'adulte en position d'autorité. Je suis l'adulte sur qui ils peuvent compter et qu'ils ont peur de décevoir. Parfois ils font des trucs que personnellement, en tant qu'humain, je trouve décevant, parce que tu veux toujours le bien pour les gens avec qui tu développes un lien. Je leur dis : «C'est correct, mais faut juste que tu comprennes pourquoi t'as fait ça, et pourquoi tu voudrais pas que ça se reproduise. C'est quoi la leçon ? Toi qu'est-ce que

7 La démocratie culturelle est un modèle de politique culturelle d'origine française né dans les années 1980, sous l'impulsion du ministre de la culture Jack Lang. Les pratiques amateurs se placent au centre de la réflexion. Cette période a été précédée par la démocratisation culturelle, inspirée de l'éducation populaire et initiée par André Malraux, toujours en France dans les années 1960. Elle s'est incarnée, entre autres, par la création d'un réseau de maisons de la culture. Il s'agissait de rendre accessibles les œuvres artistiques canonisées par un processus de décentralisation.

8 Youssoupha est un rappeur français dont l'écriture, comme celle d'Oxmo Puccino, se rattache au courant du rap conscient. 
t'en penses, parce que c'est pas important moi ce que j'en pense. » Mon objectif c'est vraiment de développer l'esprit critique. Ultimement, c'est ça.

E. F. : Présente-nous le projet Samplicité volontaire.

к. т. : Ça, c'est un projet que j'ai rédigé en partenariat avec Exeko ${ }^{9}$, ils avaient un appel de projets de médiation et j'ai proposé Samplicité volontaire. Dans le fond, le but est vraiment d'engager les gens dans une démarche musicale en direct. Donc moi j'arrivais avec des vinyles, du matériel pour faire de la musique et j'avais un micro pour enregistrer les gens. Les gens pouvaient venir, regarder dans les vinyles et s'inspirer de ce qu'il y avait là pour que je puisse échantillonner et faire la musique. Les gens pouvaient enregistrer quelque chose, lire un truc, chanter. Ils pouvaient dire quelque chose, taper des mains. Des fois, on avait des instruments à portée de mains. C'était intéressant parce que ça engageait vraiment un dialogue avec les gens, à partir de toute sorte de médium. Par exemple, à un moment donné, il y avait un gars qui venait de sortir de prison, $\mathrm{j}$ 'avais un vinyle de Chris de Burgh ${ }^{10}$, puis il me disait qu'il y avait une chanson là-dessus qui l'avait vraiment aidé à endurer son séjour en prison. Pour te donner un autre exemple, il y avait une dame ukrainienne. Parce que j'avais un vinyle de musique ukrainien de son enfance, elle était là : "Oh my God! Comment ça se fait que vous avez ça ? » Puis là, elle m'expliquait dans quel contexte elle écoutait ça quand elle était jeune, quand il y avait des fêtes, parce qu'il y avait des grandes réunions familiales ; donc l'idée c'est vraiment de créer un espace où les gens pouvaient communiquer, échanger, dialoguer autour de la musique et, s'ils le veulent, participer.

Le but, c'était vraiment de créer un espace où ils peuvent parler, juste viber, essayer de partager un intérêt commun. C'est drôle, parce que parfois il y avait vraiment des gens qui étaient des musiciens, des gens qui étaient fans de musique, des gens qui étaient là en tant qu'observateurs ou observatrices, donc c'était super intéressant de voir la mixité de tout ce monde-là. Voir comment ces personnes qui viennent vraiment d'horizons différents échangent. La musique, je trouve que c'est quelque chose de très rassembleur ; le but du projet c'était ça : de montrer que tu pouvais rassembler les individus autour de la musique sans nécessairement avoir besoin d'être musicien.

Si les formations dédiées à la médiation culturelle ont fait leur apparition dans certaines universités francophones (Paris, Lausanne, Montréal), il est courant de rencontrer des médiateurs et médiatrices qui se sont formé·e·s sur le terrain ou à travers des expériences issues d'autres champs disciplinaires ${ }^{11}$, comme c'est le cas ici avec l'intervention sociale pour Kenny Thomas. Le mot médiation culturelle est venu s'apposer à une pratique déjà existante depuis plusieurs décennies.

\footnotetext{
9 Exeko est un organisme d'innovation sociale basé à Montréal, créé en 2006. Il utilise la créativité intellectuelle et artistique en la mettant au service d'une transformation sociale inclusive et émancipatrice. Pour plus d'informations, voir le site Internet d'Exeko : https://exeko.org/fr/a-propos, consulté le 19 janvier 2020.

10 Chris de Burgh est un chanteur et musicien de folk britannico-irlandais.

11 Voir les autres entrevues de la revue.
} 
PARCoURS DE MÉdIATEUR : FORMATION, APPRENTISSAGE, TRANSMISSION

E. F. : Tu as un $\mathrm{DEC}^{12}$ en travail social. Comment en es-tu venu à t'intéresser à ce champ jusqu'à te former pour devenir intervenant social ?

к. т. : Quand j'étais jeune, à 15 ans, je fréquentais un organisme communautaire, qui s'appelle le Café Jeunesse Multiculture ${ }^{13}$ à Montréal-Nord. J'ai fini par y travailler, c'était mon premier emploi. Mon père avait fait un AVC et ça l'avait laissé paralysé ; je n'étais vraiment pas dans une bonne phase à l'époque. Là, y'a un des intervenants qui me dit : «T'as pas l'air de feeler... Ken, je comprends ce que tu vis, mais dans la vie il y a toujours le choix de définir l'importance que les choses ont, ou tu laisses les choses te définir toi. » Puis je ne sais pas, ça a été comme un moment de Luke qui rencontre Obi-Wan et à ce moment-là ; je me suis dit : " OK, moi je veux faire ça dans ma vie. Ce que lui m'a donné à ce moment-là, je veux pouvoir le transmettre aux autres. »

La figure du mentor est illustrée par la référence de Kenny Thomas à la relation interpersonnelle qui lie le maître jedi Obi-Wan Kenobi à son apprenti Luke Skywalker, deux personnages qui existent dans l'univers de la saga Star Wars, monument cinématographique de la culture pop. Elle est importante à mettre en lumière, car elle représente la dimension " informelle " de la formation de Kenny Thomas. C'est en observant, en participant, et surtout en étant présent sur le terrain qu'il a appris son métier, au contact d'autres intervenant·e·s capables de transmettre leurs connaissances. Le processus d'apprentissage du métier est en constant renouvellement et Kenny Thomas continue de se former pour s'améliorer et pour pouvoir former et accompagner la relève dans le même temps.

K. т. : Concernant l'intervention, j'ai eu des mentors ; au niveau de la médiation, c'est venu de façon tout à fait naturelle. Dans la musique, dans la culture urbaine, même dans le break dance... tout le monde à un mentor, tout le monde a quelqu'un qui lui a montré les moves, les façons de mieux donner le flow. Il y a cette espèce de mentorat-là qui fait partie de l'ADN de la culture hip-hop, de la culture urbaine, je pense. Après, je me suis formé sur le terrain, j'ai fait de l'intervention de rue, avec des jeunes. J'ai même été aux États-Unis dans certains quartiers défavorisés faire du bénévolat il y a longtemps : t'apprends. Qu'il s'agisse de l'intervention ou de la médiation, selon moi il faut que tu puisses développer une approche intégrée et humaniste.

E. F. : Es-tu devenu le mentor de quelqu'un ? Tu es venu présenter tes projets aux étudiant·es du D.E.S.s. en médiation de la musique de l'Université de Montréal. Ici, tu acceptes de répondre à nos questions ; on ressent l'envie de partager ce que tu fais. En quelle mesure entres-tu dans une démarche de transmission auprès de tes pairs?

12 Le DEC est un diplôme d'études collégiales, préuniversitaire, obtenu au cégep. Ce serait l'équivalent du baccalauréat français.

13 Le cJM est un organisme à but non lucratif qui offre un lieu d'échange et des activités culturelles et artistiques pour les jeunes de 13 à 30 ans. 
K. T. : Partout où j'ai travaillé, j'ai toujours été celui qui accueillait les stagiaires. Comme tu sais, en deuxième année de travail social ou troisième année d'éducation spécialisée, c'est important d'avoir des mentors. L'école c'est un drôle de concept quand tu y penses : on te dit toute ta vie ce que tu dois faire, puis à un moment donné tu te spécialises dans quelque chose et puis soudainement c'est oK, vas-y, débrouille-toi. J'avais cette discussion-là avec mes jeunes. Y'en a qui étaient rendus au secondaire $5^{14}$, qui me disaient : "Tu sais j'ai rempli ma demande au cégep ${ }^{15}$, j'ai été accepté et là j'ai l'impression - j'ai trouvé ça vraiment brillant comme façon de le verbaliser -, j'ai l'impression d'attendre que ma vie commence. " C'est puissant comme image. On veut tellement encourager la relève, on veut que les gens aillent à l'école, qu'ils se spécialisent, mais il faut aussi leur donner une chance sur le terrain de voir ce qu'ils valent. Je comprends que du point de vue du gestionnaire, c'est une tâche de plus, mais il faut, surtout en intervention jeunesse, former la relève. J'ai eu des mentors assez solides au niveau du travail avec les jeunes qui vivent dans des dynamiques de rue, criminalité, qui viennent de familles nouvellement arrivées ou économiquement très précaires. Ça a été une bonne école pour moi. J'aurais aimé ça avoir du feedback. Je suis vraiment pour former stagiaires et étudiants, qui ont toute la volonté du monde, mais si tu n'as pas le savoir-être, le savoir-faire et le savoir sur le terrain, tu peux causer plus de tort qu'autre chose aux populations avec lesquelles tu es amené à travailler. C'est important.

Dans le cadre de mon travail présentement je suis coordonnateur clinique dans un organisme qui travaille en HLM à Ahuntsic. Je supervise les interventions d'un groupe de six intervenants et moi-même. Je débusque des formations pour justement me former pour savoir comment mieux gérer une équipe, animer des rencontres, pour la prise de notes. C'est niaiseux, mais c'est des choses qui font en sorte que ça va faire de moi un meilleur employé.

E. F. : Comment ton approche a-t-elle évolué avec le temps ?

к. т. : J'ai vécu un moment marquant il y a une dizaine d'années lorsque j'ai été confronté à un choc des générations avec des jeunes qui n'avaient pas du tout les mêmes référents culturels et musicaux que moi. Je me suis dit qu'il fallait que je m'intéresse un peu plus à autre chose que ce que je connais et surtout que je devais partir de l'expertise des jeunes ${ }^{16}$.

J'ai un jeune qui m'a appelé ce matin. Il adore le groupe pNL. Je ne sais pas ce que tu penses de ce groupe, mais moi, personnellement, je suis vraiment ... Mais je me suis dit oK, je ne peux pas m'attarder au contenu et comprendre aussi que différentes populations vont s'intéresser à différents éléments d'un même contenu.

14 En secondaire 5 les élèves ont 16-17 ans.

15 Au cégep on obtient un diplôme d'études collégiales (DEC) à l'âge de 18-19 ans, ce qui représente en moyenne deux ans d'étude.

16 Ici, nous constatons que la pratique de Kenny Thomas s'horizontalise de plus en plus, en prenant en compte l'expertise des jeunes et leurs goûts musicaux, comme souligné précédemment à la note de bas de page $\mathrm{n}^{\circ} 7$. 
Me concernant, ce sont les mots. Si tu manies bien les mots, moi j'adore ça. J'adore Oxmo, Akhenaton, pour ça, Youssoupha aussi, Grand Corps Malade ; tu sais les gars manient bien les mots, tout est réfléchi, calculé. Tu ne peux honnêtement pas dire ça de PNL. Mais je me suis dit OK, ce jeune-là il s'intéresse à ce duo pour une raison que je ne comprends pas. Il m'a dit : "Ken, il faut que tu écoutes ce groupe-là ». Un matin j'ai écouté trois albums de PNL, en boucle. Et c'est là que j'ai compris que c'était zéro le contenu, zéro la performance vocale ; c'est vraiment ce que les gars évoquent. On aime beaucoup les histoires, tu sais un peu comme Steve Jobs qui avait rien, l'histoire du underdog ${ }^{17}$, et là je me suis dit : "Ce jeune-là, quand tu te rends compte qu'il vit dans une famille où il a une petite sœur autiste dont il doit s'occuper, il n'y a pas beaucoup d'argent, il a commencé à vendre de la drogue pour subvenir à ses besoins... » Tu te dis : « OK, lui avait vraiment besoin d'un modèle de réussite, qui vient du même milieu que lui. » À partir de là je pouvais vraiment écouter PNL parce que je comprenais que ce n'était pas tant le contenu. Tu sais il y en a qui ont des coachs de vie ; je pense que PNL c'est comme un peu son coach de vie à lui, si je dois vulgariser. Et pour qu'il y ait une médiation, il faut que tu rejoignes deux pôles, deux univers. L'un pouvant être le tien, ou deux univers qui ne t'incluent pas. Il faut que tu puisses être en mesure de faire le travail pour créer le pont.

La posture de médiateur de Kenny Thomas est horizontale. Il s'efface pour devenir un initiateur d'échanges et un facilitateur. Pour cette raison, il se concentre sur les goûts musicaux et les sensibilités des participant·e·s, qui deviennent le point de départ. Cette démarche nous renvoie à l'idée de " prise » sur la musique, concept développé par Christian Bessy et Francis Chateauraynaud ${ }^{18}$ et repris ensuite par Antoine Hennion dans ses écrits sur l'amateur de musique ${ }^{19}$. Hennion avance l'idée qu'une pochette de disque en tête de gondole dans un magasin est un dispositif de médiation qui permet une prise sur la musique, comme une porte d'entrée. Pour filer la métaphore, Kenny Thomas se place derrière les portes ouvertes par les participant·e·s et non l'inverse.

E. F. : Donc ta définition de la médiation c'est ça, c'est faire le pont ?

к. т. : Oui. C'est une vision qui a beaucoup été influencée par le travail de rue, je te dirais là, c'est vraiment beaucoup de rejoindre les gens en rupture sociale, donc autant les gens en situation d'itinérance, que les travailleur·euse's du sexe, que les jeunes membres de gang de rue. Il faut être patient, disponible, et il faut être avenant. J'essaye d'être et d'avoir cette approche-là dans tout ce que je fais, dans la vie aussi.

17 L'outsider, l'opprimé, le perdant probable. Source de la traduction : https://www.wordreference. com/enfr/underdog. Pour aller plus loin sur le concept d'underdog, voir : Danny Miller et Isabelle Len Breton-Miller (2017), "Les underdog entrepreneurs. Quand l'adversité crée des bâtisseurs », Gestion, vol. 42, $\mathrm{n}^{\circ}$ 2, p. 34-36, https://www.cairn.info/revue-gestion-2017-2-page-34.htm, consulté le 23 avril 2020.

18 Christian Bessy et Francis Chateauraynaud (2014), Experts et faussaires. Pour une sociologie de la perception, Paris, Éditions Pétra.

19 Antoine Hennion, Sophie Maisonneuve et Émilie Gomart (2000), Figures de l'amateur. Formes objets et pratiques de l'amour de la musique aujourd'hui, Paris, La Documentation française. 
Surtout au niveau du travail, comme avec Samplicité volontaire. Je n'étais jamais rentré en contact avec des populations autochtones itinérantes et c'est en parlant avec des médiateurs d'Exeko que je me suis rendu compte qu'il y a vraiment beaucoup de besoins pour ces populations-là. Mais on n'en parle pas. Disons qu'au Québec on ne parle jamais des populations autochtones comme une population légitime qui a des réalités, qu'il faut aider. On en parle souvent comme de la vermine un peu avec ce discours : "Eux autres ils sont là aussi. » Donc le projet m'a vraiment ouvert à cette réalité-là. Sans vouloir sauver le monde, il faut que t'aies un peu cette espèce d'ouverture, de patience et de posture ; " je suis étranger dans un milieu et je veux juste comprendre ce milieu-là » et vouloir créer des liens dans ce milieu.

E. F. : Un des objectifs principaux de la médiation serait alors de créer du lien social?

к. т. : Oui, exact, les gens ne se parlent pas assez. On pense toujours que le problème des autres n'est jamais notre problème, alors que c'est pas le cas. Il faut ajouter l'idée que de nos jours, on est tellement isolés, encabanés dans nos affaires, dans notre téléphone, dans nos trucs, dans nos prêts et bourses... C'est vraiment légitime et compréhensible, mais il y a des gens qui vivent des réalités difficiles et on est dans une époque... J'ai une amie qui appelle ça « la dictature du bonheur» : il faut toujours que ça ait l'air nickel, tout ce qu'on fait. Maintenant c'est ça, on veut que tout le monde aille bien, on ne veut pas nécessairement être partie prenante de cette démarche-là. Je pense que c'est ça aussi, le travail de la rue, dans les HLM. Ça m'a fait comprendre qu'il n'y a rien de mauvais rien de bon, tout est la vie.

Je pense que j'en parlais avec des proches, en 1997. J'ai acheté Opera Puccino, premier album d'Oxmo Puccino, puis là, je me rappelle, j'avais un de mes amis qui avait des frères plus vieux qui m’ont dit : "Ah t'aimes ça, bah il faut que t'écoutes ça ", et il me sort une cassette, et je passe toute une soirée à s'apprendre des choses, pour moi c'est comme super important de ne pas être dans une posture " ah moi je connais plus de trucs que toi ", mais plutôt de partager. Tu sais, derrière la médiation et Samplicité volontaire, l'idée, c'est vraiment l'échange.

Grâce à cette anecdote, nous comprenons qu'il est courant d'être l'intermédiaire entre un objet artistique et une personne, d'une certaine façon de faire de la médiation. Elle est présente dans les institutions culturelles, mais aussi dans les conversations les plus anodines. Discours, pratique, outil... son objectif principal reste, comme 1'a très bien dit Kenny Thomas, l'échange, le partage et la création de lien social. 